\title{
LATE ONSET OF ASPERGILLUS AORTITIS PRESENTING AS FEMORAL ARTERY EMBOLISM FOLLOWING CORONARY ARTERY BYPASS GRAFT SURGERY
}

\section{S. Oaleed Noordally ${ }^{1}$, Schoeb Sohawon ${ }^{2}$, David De Bels ${ }^{1}$, Ruth Duttmann ${ }^{3}$, Philippe Gottignies ${ }^{1}$, Jacques Devriendt}

Department of Critical Care Medicine, Brugmann University Hospital, Free University of Brussels, Brussels, Belgium¹; Department of Surgery, Erasme Academic Hospital, Free University of Brussels, Brussels, Belgium²; Department of Pathology, Brugmann University Hospital, Free University of Brussels, Brussels, Belgium ${ }^{3}$

Summary: Aspergillus sp. are ubiquitous mould infections and in most patients, the source is presumed to be air-borne infections during surgical procedures. Prevention of these infections requires special attention of ventilation systems in operating rooms. Post-operative aspergillosis occurs mainly in immunocompromised patients as well as those who receive corticosteroids temporarily. We report a case of a 71-year-old immunocompromised patient who developed multiple lower limb embolisms due to Aspergillus niger originating from an aortitis of the ascending aorta nine months following coronary artery bypass graft (CABG) surgery.

Key words: Aspergillus; Aortitis; Cardiac surgery; Embolism; Amphotericin B.

\section{Case history}

A 71-year-old woman was admitted to our emergency department for an acute onset of a right lower limb pain. Her past medical history revealed essential hypertension, hypercholesterolemia, chronic renal failure, monoclonal gammapathy, idiopathic thrombocytopenic purpura and prophylactic splenectomy. Nine months prior to her admission, she had an aortic valve replacement and coronary artery bypass graft surgery. Her medications consisted of Aspirin $80 \mathrm{mg}$ o.d., Furosemide $20 \mathrm{mg}$ o.d., and Spironolactone $50 \mathrm{mg}$ o.d. On admission, her blood pressure was $121 / 65$, ECG tracing was 76/min and sinus, and had a pale, cold, pulseless right lower limb. Pathological blood results were: CRP $4.3 \mathrm{mg} / \mathrm{dL}(\mathrm{N}<1)$, hemoglobin of $8.8 \mathrm{~g} / \mathrm{dL}$ (N: $12-16 \mathrm{~g} / \mathrm{dL})$, a hematocrit of $27.6 \%(\mathrm{~N}: 35-47 \%$ ), a platelet count of $40,000 / \mu \mathrm{L}(\mathrm{N}: 150,000-440,000 / \mu \mathrm{L})$ and a white blood cell count of $14,700 / \mu \mathrm{L}(\mathrm{N}: 3500-11,000 / \mu \mathrm{L})$.

An Angio-CT scan of her right lower limb showed multiple emboli in her right superficial and deep femoral arteries as well as in her right popliteal and peroneal arteries and underwent successful Fogarty intervention. Femoral embolectomy specimens at histopathology showed septate dichotomously branching hyphae of Aspergillus niger (Fig. 1). Serum Aspergillus antigen index was $5.76(\mathrm{~N}<0.5)$ while blood cultures were negative.

A transesophageal echocardiography revealed a vegetative mass above her normal Carpentier-Edwards aortic prosthesis, without valvular leak (Fig. 2). She underwent a composite graft replacement of the aortic valve (CarpentierEdwards by a St. Jude prosthesis), aortic root and ascending aorta, with re-implantation of the coronary arteries into the graft (Bentall procedure). The mycotic mass was removed en bloc as well as part of the ascending aortic wall and sent for histopathological examination which confirmed Aspergillus niger aortitis (Fig. 3). She responded well to intravenous Amphotericin B $1 \mathrm{mg} / \mathrm{kg}$ and left the intensive care department 8 days later. Unfortunately, 6 days after leaving the

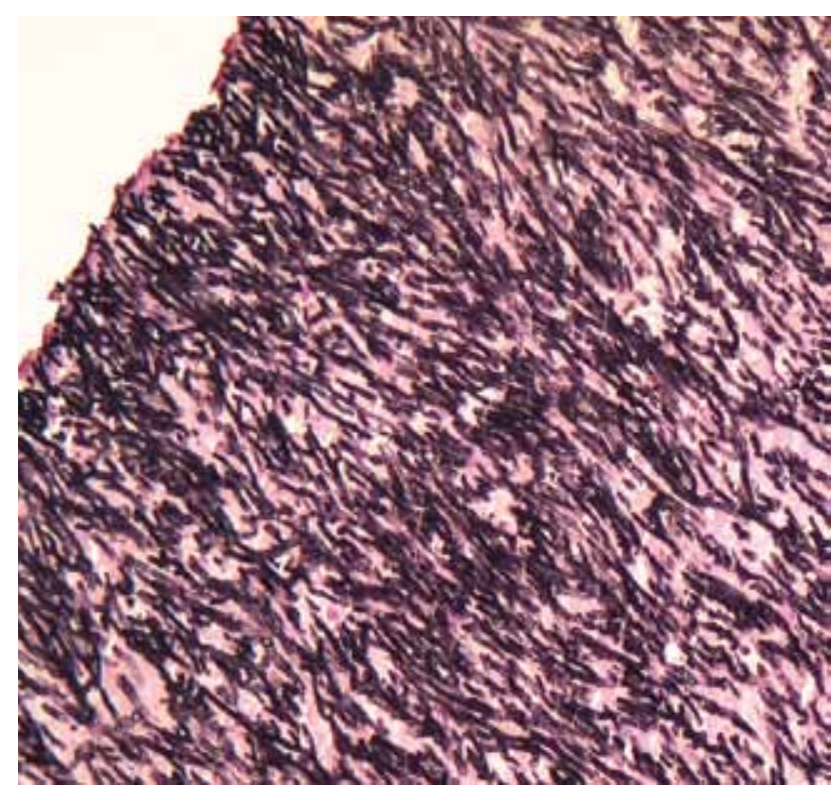

Fig. 1: Aspergillus in thrombus: parallel hyphae with septa (Grocott stain, $\times 200$ ) 


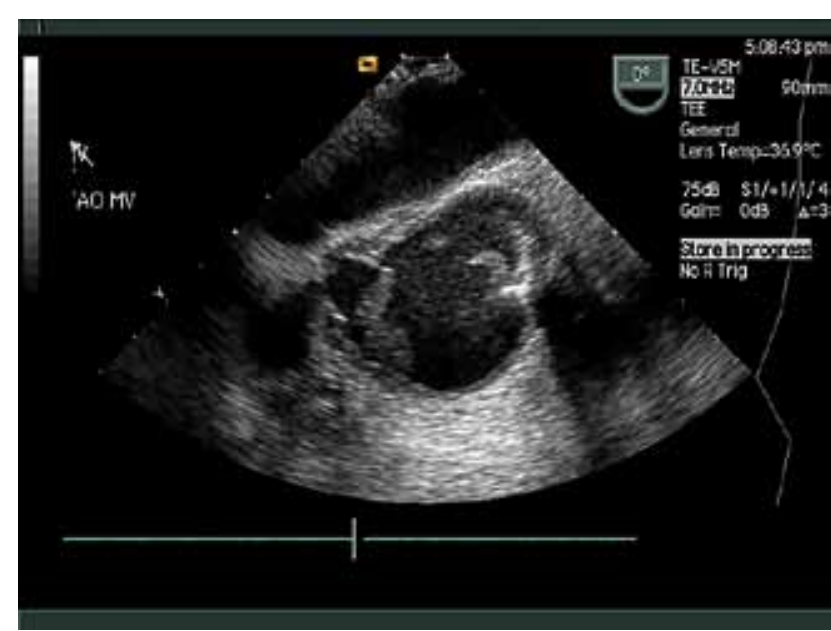

Fig. 2: Transesophageal echocardiography showing a bilobar supravalvular mass $(25 \times 13 \mathrm{~mm})$ above the coronary sinus with thickening of the posterior aortic wall

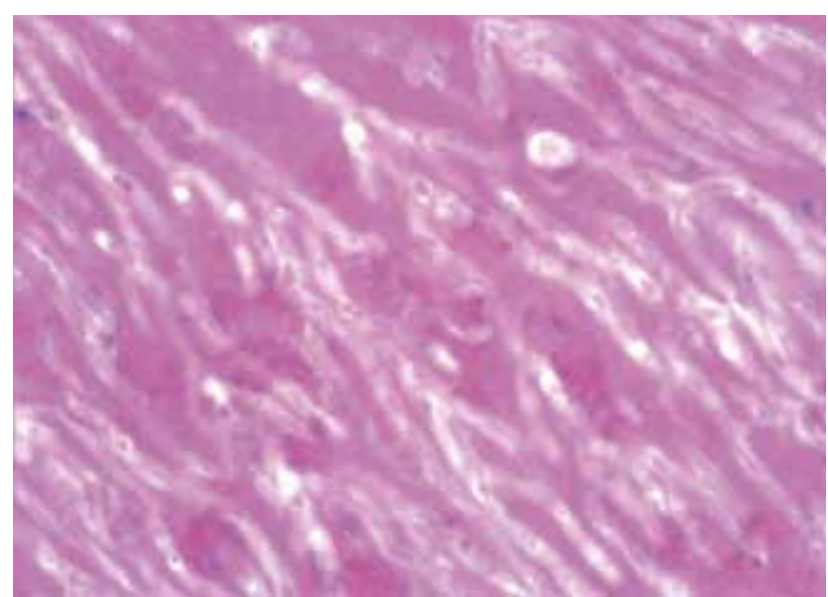

Fig. 3: Dichotomous branching, hyphae not taking PASstain (PAS stain, $\times 400$ )

intensive care unit, while she was waiting in the radiology department for a routine chest X-ray, she had a sudden cardiac arrest and died.

\section{Discussion}

We report the first case of post operative Aspergillus niger aortitis in an immunocompromised patient presenting as femoral artery embolism. Verghese et al. reported two cases of post operative endocarditis due to Aspergillus terreus and A. flavus presenting as femoral artery embolism (1). Furthermore, Sanchez-Recalde et al. reported a delayed presentation of aspergillosis aortitis of 4 to 24 months post cardiac surgery with an incidence of $0.085 \%$ of aspergillus aortitis (2). Our patient presented multiple femoral embolisms due to an ascending aortitis with concentric thickening of the aortic walls colonized by A. niger nine months following CABG surgery. The aortic valve prosthesis was free of vegetation although fungal endocarditis tends to affect mostly aortic valve prosthesis.

We considered the patient to be at risk as she has low grade monoclonal gammapathy and had prophylactic splenectomy for idiopathic thrombocytopenic purpura 20 years ago. Damage to the aortic wall during aortic cannulation at the time of aortic surgery is recognized as a risk factor for intra-operative infection, as a result of contamination with airborne fungal spores. Laminar airflow ventilation systems reduce the risks of such contaminations.

The diagnosis of aortitis was made on the basis of culture of the embolectomy specimen. The diagnosis of Aspergillus aortitis is difficult because of the absence of endocarditis vegetation. This condition is better detected by transesophageal rather than transthoracic echocardiography, with magnetic resonance imaging and aortic angiography being useful adjuncts (2). Pasqualotto et al., in a review of patients with aortitis due to Aspergillosis aortitis following cardiac surgery reported an overall mortality rate of $92.7 \%$ (3). Moreover, in their study, $11.2 \%$ of Aspergillus aortitis were due to the niger species. Amphotericin B remains the treatment of choice while in cases of resistant strains, voriconazole is the antifungal of choice.

\section{Conclusion}

Distal embolisms in immunosuppressed post operative cardiac surgery patients with low grade fever should be investigated for mould aortitis or endocarditis. Nonetheless, investigations of patients and ventilatory systems at surgery during the time frame of the surgical procedure of our patient failed to detect any fungal contaminants.

\section{References}

1. Verghese S, Maria CF, Mullaseri AS, Asha M, Padmaja P, Padhye AA. Aspergillus endocarditis presenting as femoral artery embolism. Mycoses 2004; 47: 252-256.

2. Sanchez-Recalde A, Mate I, Merino JL, Simon RS, Sobrino JA. Aspergillus aortitis after cardiac surgery. J Am Coll Cardiol 2003; 41: 152-156.

3. Pasqualotto AC, Denning DW. Post-operative aspergillosis. Clin Microbiol Infect 2006; 12: 1060-1076.

\section{Corresponding author:}

Dr S. Oaleed Noordally, Division of Critical Care Medicine, Brugmann University Hospital, Free University of Brussels, Brussels, Belgium; e-mail: sheikoaleed.noordally@chu-brugmann.be 